\title{
Design of a compact astrometric instrument for the GAME mission
}

\author{
D. Loreggia, M. Gai, A. Vecchiato, D. Gardiol, S. Ligori \\ and M. G. Lattanzi \\ All Authors are from INAF - Astronomical Observatory of Turin, St. Osservatorio, 20, 10025 \\ Pino Torinese, Torino, Italy. \\ email: loreggia@oato.inaf.it
}

\begin{abstract}
We present the design of a Fizeau interferometer to be implemented for the GAME mission. The aim is to measure the PPN $\gamma$ parameter with the same technique used for the first time by Dyson, Eddington et al., but at a $10^{-6}$ accuracy level. GAME will observe about $10^{6}$ sufficiently bright stars at about $2^{\circ}$ from the Sun. A dedicated space mission has the advantage of observing the light bending without waiting for an eclipse.
\end{abstract}

Keywords. gravitational lensing, space vehicles, instrumentation: interferometers, techniques: high angular resolution

\section{Introduction}

The Gamma Astrometric Mission Experiment - GAME (Vecchiato, et al.2008) will observe two symmetric fields, each about $2^{\circ}$ from the Sun. The relative position of the targets is measured again 6 months later, without the Sun in between, giving a direct estimation of the light bending and the $\gamma$ PPN parameter with an expected accuracy of $10^{-6}$. Hereafter we briefly discuss a possible telescope design, preserving some requirements for a small mission.

\section{Optical design}

A 5-aperture Fizeau interferometer design is well suited for our propose of merging good performance with some geometrical constraints such as a distance between the main mirrors not larger that $1.5 \mathrm{~m}$, the size of the pupil compatible with diffraction limited (DL) images and with the size and the geometry of the interferometric apertures. These requirements must match the best sampling condition of the fringes (Loreggia, et al.), assuming a pixel size of $15 \mu \mathrm{m}$ in the high resolution direction (HRD) and asking for a baseline between each couple of apertures $\mathrm{B}=2 \mathrm{~L}$, where $\mathrm{L}$ the aperture side in HRD. The aperture area dimensioning sets directly all the instrument parameters and is driven by the photons collecting needs and the final encumber limitations. At $\lambda_{r e f}=650 \mathrm{~nm}$, the best solution is obtained with apertures of $40 \times 220 \mathrm{~mm}$ over a pupil of $660 \mathrm{~mm}$, giving an $\mathrm{EFL}=20600 \mathrm{~mm}$ and a total baseline (between the outer apertures) $\mathrm{B}_{t}=320 \mathrm{~mm}$, as shown in Fig.1.

GAME will look at two lines of sight very close to the Sun, which combination and folding is implemented by mean of a beam combiner in front of the primary mirror. A baffling array is needed and is under study; we tested that looking at about $2^{\circ}$ from the Sun, a typical baffling length may be $\sim 4.2 \mathrm{~m}$, which can be achieved with the folded configuration presented. 

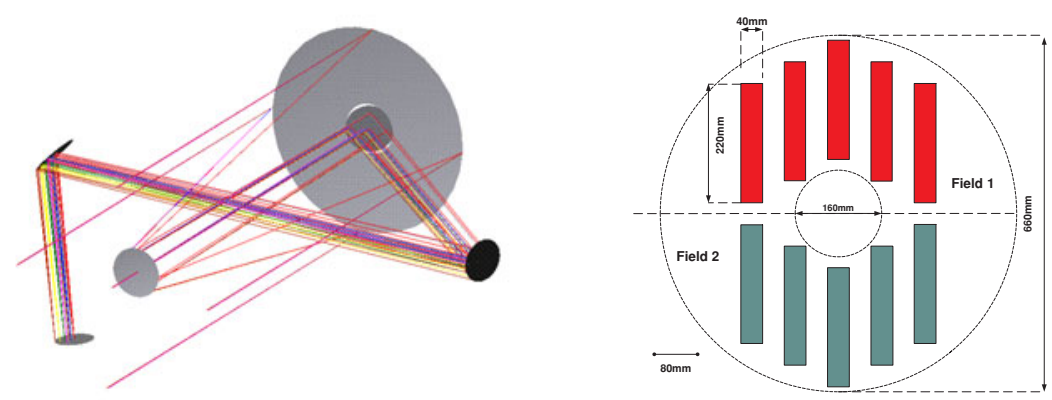

Figure 1. Telescope 3D layout (left); 5 apertures interferometric mask (right)

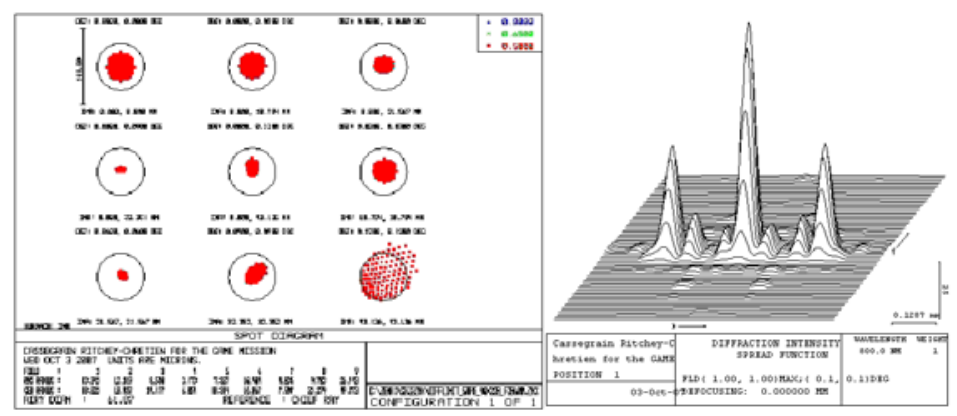

Figure 2. Spot diagram for the full aperture: circle dimension gives the diffraction limit equal to $50 \mu \mathrm{m}$ (left). Fringe pattern for the representative field $(0.12,0.12) \mathrm{deg}$ (right)

\section{Results}

In Fig (2) the spot diagrams of the full aperture are given for some reference fields over a FOV of 7'x7'. The outer field $(0.12,0.12) \mathrm{deg}$ is not DL but when the interferometric mask is applied the expected reducing of aberration makes the fringes quality good over the whole FOV, with a quite constant $\mathrm{V}=\mathbf{0 . 9 5}$. The accuracy of an astrometric measurement is linked to instrument gain parameter, the SNR and the $X_{R M S}$ equivalent aperture of the interferometer. It can be shown that for the 5 apertures interferometer geometry we are considering, $X_{R M S}=114 \mathrm{~mm}$. Assuming to work in the $\mathrm{V}$ band with a sufficiently narrow FWHM and an exposure of 100 sec, the SNR is equal to 385 (no extinction, instrument transmission $\mathrm{T}$ and $\mathrm{QE}=90 \%$ ) and we get an accuracy on the single measurement $\sigma=\mathbf{0 . 5 8 m a s}$. This precision scales with the magnitude approximatively as $10^{0.2\left(m-m_{0}\right)}$. At $2^{\circ}$ from the Sun the light deflection is $\Delta \alpha \cong 0.2$ " and the error $\sigma_{\Delta \alpha}$ is related to the accuracy of the $\gamma$ parameter estimation by (Vecchiato, et al.2008):

$$
\frac{\sigma_{\Delta \alpha}}{\Delta \alpha} \cong \frac{\sigma_{\gamma}}{2 \gamma} \quad \Rightarrow \quad \sigma_{\gamma}=2 \gamma \sigma_{\Delta \alpha} / \Delta \alpha=\sigma_{\Delta \alpha} \cdot \mathbf{1 0}^{-\mathbf{2}}
$$

This means the need to observe at least $10^{6}$ objects to have the expected final accuracy of $10^{-6}$, an accessible result from this mission (Vecchiato, et al.2008).

\section{References}

Loreggia, D., Gardiol, D., Gai, M., Lattanzi, M. G., \& Busonero, D. 2004, Apl.Opt Vol.53 No. 4., 721-28;

Vecchiato, A., Lattanzi, M. G., Gai, M., \& Morbidelli, R., Gamma Astrometric Mission Experiment, Proc. IAU Symposium No.248, in this volume, p.290 